\title{
Slave Bosons in Radial Gauge: the Correct Functional Integral Representation and Inclusion of Non-Local Interactions
}

\author{
Raymond Frésard ${ }^{1, a *}$ and Thilo Kopp ${ }^{2 \dagger}$ \\ ${ }^{1}$ Institut de Physique, Université de Neuchâtel, A.-L. Breguet 1, 2000 Neuchâtel, Switzerland \\ ${ }^{2}$ Experimentalphysik VI, Elektronische Korrelationen und Magnetismus, Universität Augsburg, D-86135 Augsburg, Germany
}

\begin{abstract}
We introduce a new path integral representation for slave bosons in the radial gauge which is valid beyond the conventional fluctuation corrections to a mean-field solution. For electronic lattice models, defined on the constrained Fock space with no double occupancy, all phase fluctuations of the slave particles can be gauged away if the Lagrange multipliers which enforce the constraint on each lattice site are promoted to time-dependent fields. Consequently, only the amplitude (radial part) of the slave boson fields survives. It has the special property that it is equal to its square in the physical subspace. This renders the functional integral for the radial field Gaussian, even when nonlocal Coulomb-type interactions are included. We propose i) a continuum integral representation for the set-up of further approximation schemes, and ii) a discrete representation with an Ising-like radial variable, valid for long-ranged interactions as well. The latter scheme can be taken as a starting point for numerical evaluations.
\end{abstract}

*Email: Raymond.Fresard@ismra.fr

${ }^{\dagger}$ Email: Thilo.Kopp@physik.uni-augsburg.de 


\section{INTRODUCTION}

The significance of strong electronic correlations in metals has long been acknowledged when intractable enigmas of solid state physics such as itinerant magnetism, the correlation-induced metal-insulator transition, unconventional superconductivity, or spin liquid states and non-Fermi liquid behavior of electronic excitations were embraced. The scope of the "strongly correlated physics" is nowadays enormous; it extends to the fractional quantum Hall effect and high- $T_{c}$ superconductivity, and also in mesoscopic systems an increasing number of investigations take this route since certain scenarios with strong local interactions can be modeled more directly in experiments on a mesoscopic scale. The efforts to fathom the underlying mechanisms resulted only in partial success. Especially, models with strong local interactions of itinerant electrons resisted a thorough understanding beyond certain limiting, and often unphysical, cases.

The celebrated Hubbard model epitomizes the two opposing characters of narrow band electrons: electron hopping (with energy scale $t$ ) supports the itinerant character, suitably expressed in momentum space, and favors a metallic character. In contrast to this, the local on-site Coulomb interaction $U$ may drive the electrons, depending on bandfilling, into a Mott insulating state, with strong magnetic correlations. Standard perturbation theory is not applicable in the transition regime (for a recent discussion see [1]), and whether it even was valid for any finite $U$ in two space dimensions [2] was a longstanding problem. On the other hand, perturbation theory from the atomic limit, with no kinetic terms, is not feasible in a straightforward manner since the tremendous degeneracy for low energy states of the fully localized limit renders the usual perturbation theory inapplicable. Furthermore, a hopping expansion cannot build on Wick's theorem since the zeroth order Hamiltonian is not bilinear in the canonical electron fields, though progress has been achieved recently [3, 何. Nonetheless, for $t \ll U$ a Schrieffer-Wolff transformation may be performed to the appropriate order in $t / U$. This may be visualized as a rotation of the states in Hilbert space such that transitions between different subspaces with fixed number of doubly occupied sites are suppressed to the corresponding order in $t / U$. Equivalently, one usually applies a canonical transformation to the Hamilton operator in order to generate an effective Hamiltonian of which the low energy part is explicit. The Hubbard model is reduced in this way [5] to a $t-J$ model (plus pair-hopping) up to linear order in $t / U$ - or to a " $t$-model" in zeroth order. The $t$-model is just the kinetic term, however it is now a correlated hopping in the sense that double occupancies are excluded. This constrained hopping can only be envisioned in real space. The $t-J$ model includes, beyond the constrained hopping, a Heisenberg spin-exchange and a short range density-density correlation term of scale $J \sim t^{2} / U$. Although all these strong coupling models present the local spin and charge excitations in an appealing way, it is still a long way to extract the true (delocalized) low-energy excitations.

In the past decades considerable effort has been invested into the construction of techniques which work in the constrained Fock space. Already Hubbard introduced projection operators onto local (site-) states with no electron $(|0\rangle)$, one electron with spin $\sigma(|\sigma\rangle)$ or double occupancy $(|2\rangle)$. These so-called Hubbard X-operators belong to a graded Lie-algebra, and a canonical many-particle Green's function formalism is not applicable (for a detailed review of functional integral representations and a suitable choice of coherent states for X-operators see ref. [6]). The evaluation scheme by Hubbard shaped the appropriate picture of lower and upper "Hubbard bands" for low and high energy (in-) coherent excitations, respectively. Yet it suffered from inconsistencies, e.g. the failure to satisfy the Luttinger theorem.

An alternative approach, which also implements a projection onto local states, is the slave boson formalism [7, 8]. Electron creation and annihilation operators are represented by composite operators whereby an electron is annihilated either by the creation of a hole site, accompanied by the annihilation of a singly occupied site with corresponding spin or the annihilation of a doubly occupied site, accompanied by the creation of a singly occupied site with opposite spin. Several choices exist how to ascribe fermionic and bosonic degrees of freedom to these operators, for a discussion see [9]. Although the operators are now canonical fermionic and bosonic operators, they live in a constrained Fock space since the sum of their respective particle numbers (the pseudo charge) has to be unity for each site. Mean field or saddle point approximations satisfy the constraint only on the average and, furthermore, lead to Bose condensation of the slave bosons [10]. Actually, the saddle point approximation becomes exact in the unphysical limit $\mathcal{N} \rightarrow \infty$, where $\mathcal{N}$ is the spin degeneracy. Corrections to the mean field result were calculated in the first order of the expansion parameter $1 / \mathcal{N}$ - the constraint is thereby replaced by a "soft constraint" with pseudo charge equal to $\mathcal{N} / 2$. For $\mathcal{N}=\infty$, the bosons condense, that is, they attain a finite complex expectation value, the square of which is the density of empty sites.

In the Barnes representation, only the charge degree of freedom is represented by the slave boson. In contrast, both spin and charge degrees of freedom are carried by bosons (and fermions) in the Kotliar and Ruckenstein representation [8], while in the spin and charge rotation invariant representation [9] they are carried by bosons only. The meanfield approach to the Kotliar and Ruckenstein representation has been quite successful when compared to numerical simulations. Indeed, the paramagnetic mean-field solution is equivalent to the Gutzwiller approximation [8]. Even 
though this is the saddle point of an action, it turns out to obey a variational principle in the limit of large spatial dimensions, where the Gutzwiller approximation and the Gutzwiller wave function are identical 11 . When the dimension is reduced, they are not known to differ markedly. Comparison of ground state energies 12 , 13., and charge structure factors 14 with Quantum Monte Carlo data show excellent agreement. A variety of saddle points have been investigated, in particular in the vicinity of the Mott transition [15] 18]. The Mott gap can be calculated as well [19], and comparison with experimental data in $\mathrm{La}_{x} \mathrm{Y}_{1-x} \mathrm{TiO}_{3}$ [20] displays a very good agreement [17]. Nevertheless the computation of fluctuations, in particular in the magnetic channel, remains problematic.

Moreover such a finite expectation value of the complex slave boson field violates Elitzur's theorem according to which, in a theory with local gauge invariance, only locally gauge invariant operators may acquire a non-zero expectation value. Correspondingly, for finite $\mathcal{N}$, the phase fluctuations of the boson field suppress the condensation. A zero expectation value of the bosonic field, however, is problematic as a starting point since the bosonic field has to connect to the number of empty sites, in the end. It was early on recognized that these difficulties are avoided in a polar decomposition where the zero mode of the phase fluctuations may be singled out and the expectation value of the radial amplitude is, in general, non-vanishing [21,22] (a more recent, detailed discussion of $1 / \mathcal{N}$-corrections in the "radial gauge" is found in the review by Arrigoni et al. [23]). Although subtleties are associated with the measure and the correct translation of the time discretized functional integral into the continuum limit version, the result in $1 / \mathcal{N}$ is not different from that of an X-operator approach 24 .

Despite the progress in the understanding of the fluctuations around the mean field saddle point, the physically most interesting case with the largest possible spin fluctuations, $\mathcal{N}=2$, remains inaccessible. Moreover, the saddle point solution is generically a Fermi liquid solution and, therefore, non-Fermi liquid behavior is necessarily nonperturbative in $1 / \mathcal{N}$. This motivated the Karlsruhe group [25 to device a slave-boson formalism which builds on canonical many-particle techniques, is locally gauge-invariant by construction and, most importantly, allows for Fermi liquid and non-Fermi liquid behavior for the considered (multi-channel) Anderson impurity problem. It permits to recover the physical quantities over the complete temperature range, from the high temperature local moment regime to the low temperature correlated many-body state. In spite of this major success of slave boson theory, it is still a formidable problem to set up a similar diagrammatic evaluation for strongly correlated itinerant electrons.

It is quite instructive to compare the implementation of the constraint in this diagrammatic formalism with that in the functional integral radial gauge representation. In the first approach, local gauge invariance is guaranteed through Ward identities in a conserving approximation. This enforces the constraint only in so far as transitions between sectors of the Fock space with different pseudo charge are suppressed during the time evolution of a considered many particle state. The projection onto the physical sector of the Fock space is achieved in a last step, when physical expectation values are calculated, using the Abrikosov procedure of sending the Lagrange multiplier of the constraint to infinity. This may be contrasted with the functional integral calculation in the radial gauge: here the phase is fixed and the amplitude of the boson will in turn strongly fluctuate. The pseudo charge conservation will not be satisfied a priori. This is precisely the reason why one has to project onto the physical subspace at each time step (and, of course, at each site) which is accomplished by promoting the Lagrange multiplier of the constraint into a field.

In this paper we reexamine functional integrals for strongly correlated electrons with $\mathcal{N}=2$ in the radial gauge. We restrain our considerations to " $U \rightarrow \infty$ ", that is the limit of strong local interaction between electrons so that the number of doubly occupied sites is zero. The Hubbard model is reduced to either the $t-$ or $t-J$ model. In this limit, the bosonic fields in radial representation reduce to their respective (real) amplitude since all time derivatives of the corresponding phases can be absorbed in a (time-dependent) Lagrange multiplier field.

It is possible to construct the functional integral either as an integration over continuous values of the boson radial amplitudes and the Lagrange multiplier, or, equivalently one can implement the constraint first and thereby reduce the integration over the slave boson amplitude to a discrete sum over the values of "hole occupancy", $\{0,1\}$. The first method is more appropriate for approximation schemes which further manipulate the path integral analytically, and the latter method is to be taken as a starting point for numerical calculations in the strong coupling limit which do not build on a Hirsch decoupling [26].

In general, there are no ab initio advantages of one functional integral representation over another representation. It depends on the approximation scheme if a concrete representation is suitable. For example, when $1 / \mathcal{N}$-fluctuations are considered the radial gauge consistently separates the zero-mode fluctuations. Moreover, for any $\mathcal{N}$, the square of the slave boson amplitude is equal to the amplitude itself since the constraint projects the amplitude onto the values $\{0,1\}$. In the radial gauge, this renders the integrations over the real fields Gaussian, albeit the bosons are still coupled to the fermionic Grassmann fields. This projection property also ensures that the measure does not produce the cumbersome logarithmic terms in the action and, more significantly, non-local density-density interactions turn out to be Gaussian terms in the functional integral. The latter is not valid in the Cartesian gauge. These observations should suffice as motivation to readdress the construction of the slave boson functional integral in the radial gauge.

In Section II we introduce the correct functional integral representation in the radial gauge, in the limit $U \rightarrow \infty$. In Section III we propose an alternative discrete representation which may serve for numerical simulations. In Section IV] 
we extend these representations to lattice models, with (possibly) long-ranged density-density interactions. Several calculations for simple models are carried out to illustrate this new method.

\section{FUNCTIONAL INTEGRAL REPRESENTATION IN THE RADIAL GAUGE.}

In any slave boson representation of strongly correlated electron systems there is a gauge symmetry group as a consequence of the redundancy of the representation of the physical electron operator. The first slave boson representation is due to Barnes [7]. It was introduced to tackle the single-impurity Anderson model. The local physical electron operator $c_{\sigma}^{\dagger}$ is, in the limit of infinitely strong on-site interaction, rewritten as a product of auxiliary fields $f_{\sigma}^{\dagger}$ and $b$ :

$$
c_{\sigma}^{\dagger}=b f_{\sigma}^{\dagger}
$$

Here $f_{\sigma}^{\dagger}$ is a doublet of canonical fermionic fields, and $b$ a canonical bosonic field. We stay in the physical sector of the Fock space provided the auxiliary fields fulfill the constraint relation:

$$
Q \equiv \sum_{\sigma} f_{\sigma}^{\dagger} f_{\sigma}+b^{\dagger} b=1
$$

In a functional integral representation of the partition sum $Z$, the constraint is enforced via the integration over a Lagrange multiplier $\lambda$ (for a standard reference see [27]):

$$
Z=\int_{-\pi / \beta}^{\pi / \beta} \frac{\beta d \lambda}{2 \pi} e^{i \beta \lambda} \int \prod_{\sigma} D\left[f_{\sigma}, f_{\sigma}^{\dagger}\right] \int D\left[b, b^{\dagger}\right] e^{-\int_{0}^{\beta} d \tau \mathcal{L}^{\prime}(\tau)}
$$

with

$$
\mathcal{L}^{\prime}(\tau)=\mathcal{L}_{f}(\tau)+\mathcal{L}_{b}(\tau)+\mathcal{L}_{t}(\tau)+\mathcal{L}_{\text {nloc }}(\tau)
$$

and

$$
\begin{aligned}
& \mathcal{L}_{f}(\tau)=\sum_{\sigma} f_{\sigma}^{\dagger}(\tau)\left(\partial_{\tau}-\mu+i \lambda\right) f_{\sigma}(\tau) \\
& \mathcal{L}_{b}(\tau)=b^{\dagger}(\tau)\left(\partial_{\tau}+i \lambda\right) b(\tau)
\end{aligned}
$$

The last two contributions to the Lagrange density are hopping of electrons $\left(\mathcal{L}_{t}\right)$ and the non-local part of the electronelectron interaction $\left(\mathcal{L}_{\text {nloc }}\right)$, respectively. The chemical potential, which fixes the average number of electrons, is $\mu$. The space dependence (lattice site index $i$ ) of the fields and of $\lambda$ is suppressed in Eq. (3), for simplicity. Since the pseudo charge $Q$, defined in Eq. (2), is a conserved quantity, the action is invariant under a group of local $U(1)$ gauge transformations [22]. In the continuum limit, it reads:

$$
\begin{aligned}
f_{\sigma}(\tau) & \rightarrow \tilde{f}_{\sigma}(\tau)=f_{\sigma}(\tau) e^{i \varphi(\tau)} \\
b(\tau) & \rightarrow \tilde{b}(\tau)=b(\tau) e^{i \varphi(\tau)} \\
\lambda & \rightarrow \tilde{\lambda}(\tau)=\lambda-\dot{\varphi}(\tau)
\end{aligned}
$$

The physical electron operators are invariant under this transformation. It is well known that $\lambda$ has to be continued into the complex plane, and the integration contour has to be shifted into the lower half-plane in order to ensure convergence of the functional integral [28]:

$$
\lambda \rightarrow \lambda-i \lambda_{0}
$$

Moreover, the phase of the bosonic field may be gauged away by promoting the constraint into a field. The remaining degree of freedom of the boson is consequently its amplitude. Having gauged away the phase of the slave boson brings us to the radial gauge.

Certainly, the expectation value of any physical observable has to be gauge invariant. In the conventional Cartesian representation — with the integration over both, the real and imaginary components of the bosonic field — the particle number of bosons (holes) is fixed within a gauge-invariant, conserving approximation 25. In the radial representation, however, the gauge is usually fixed in order to reduce the bosonic field to a real field, and the number of bosons is 
fluctuating. Only in the final integration over the Lagrange multiplier field $\lambda(\tau)$, the particle number conservation is ensured at each time step. This situation is somewhat reminiscent of the theoretical modeling of superconductivity where, in BCS theory, the phase of the condensate is fixed, but gauge invariance is restored in RPA [29]. Nonetheless the situation for superconductivity is distinct because there, a global gauge is broken, whereas here a broken local gauge has to be restored.

In a specific gauge, the correctness of the representation can be verified through the evaluation of the partition sum, and any correlation function, in the atomic limit. In this case the calculation is straightforward. For example, the partition sum reads:

$$
Z_{\text {at }}=\int_{-\pi / \beta}^{\pi / \beta} \frac{\beta d \lambda}{2 \pi} e^{\beta\left(i \lambda+\lambda_{0}\right)} Z_{f} Z_{b}=1+2 e^{\beta \mu}
$$

where $Z_{f}=\left[1+e^{-\beta\left(i \lambda+\lambda_{0}-\mu\right)}\right]^{2}$ and $Z_{b}=\left[1-e^{-\beta\left(i \lambda+\lambda_{0}\right)}\right]^{-1}$. Note that the projection onto the physical subspace $\left(\int d \lambda\right)$ follows from a contour integral in the complex $z$-plane, with $z \equiv e^{-i \beta \lambda}$, and it only picks up the residue at $z=0$.

One may try to carry out this program in the polar representation, namely by introducing the amplitude $r_{n}$ and the phase $\varphi_{n}$ of the bosonic field:

$$
\int_{-\infty}^{\infty} \int_{-\infty}^{\infty} \frac{d b_{n}^{\prime} d b_{n}^{\prime \prime}}{\pi} \rightarrow \int_{0}^{\infty} d\left(r_{n}^{2}\right) \int_{0}^{2 \pi} \frac{d \varphi_{n}}{2 \pi}
$$

The bosonic part of the partition sum can be calculated [30], and the result is again correctly $Z_{b}$. Nevertheless, in this way one does not promote $\lambda$ into a field, and the phases $\varphi_{n}$ are not gauged away and, more importantly, the action to lowest order in the time step, is not a bilinear form in $r$ and $\varphi$ [30].

We now start to set up the path integral representation in the "radial gauge". In the first place we observe that, contrary to speculations that the radial representation simply results from a straightforward coordinate transformation, it needs to be set up on a discretized time mesh from the beginning. A translation which respects the requirement that the integrals converge, irrespective of the sequence of the various integrations, is the following:

$$
Z_{\text {at }}=\lim _{N \rightarrow \infty} \lim _{\epsilon \rightarrow 0^{+}} \lim _{\Omega \rightarrow \infty} \int_{0}^{\infty} D\left(r_{n}^{2}\right) \int_{-\Omega}^{\Omega} D\left[\frac{\delta \cdot d \lambda_{n}}{2 \pi}\right] \int \prod_{\sigma} D\left[f_{n, \sigma}, f_{n, \sigma}^{\dagger}\right] e^{-S_{f}-S_{b}+\delta \sum_{n}\left(i \lambda_{n}+\lambda_{0}\right)}
$$

where

$$
\begin{aligned}
S_{f} & =\sum_{n=1}^{N} \sum_{\sigma} f_{n, \sigma}^{\dagger}\left[f_{n, \sigma}-f_{n-1, \sigma}\left(1-\delta\left(i \lambda_{n}+\lambda_{0}-\mu\right)\right)\right] \\
S_{b} & =\delta \sum_{n=1}^{N}\left(r_{n}^{2}-\epsilon\right)\left(i \lambda_{n}+\lambda_{0}\right)
\end{aligned}
$$

and $\delta=\beta / N$ with $N$ the number of time steps.

We proceed with the evaluation of the atomic partition sum, step by step, in order to convince the reader of the correctness of this representation: integration over the Grassmann variables $\left[f_{\sigma}, f_{\sigma}^{\dagger}\right]$ yields (we implicitly understand that the limit $N \rightarrow \infty$ has to be taken at the end of the calculation):

$$
\begin{aligned}
Z_{\mathrm{at}} & =\lim _{\epsilon \rightarrow 0^{+}} \lim _{\Omega \rightarrow \infty}\left(\prod_{n=1}^{N} \int_{-\Omega}^{\Omega} \frac{\delta d \lambda_{n}}{2 \pi} \int_{0}^{\infty} d\left(r_{n}^{2}\right) e^{-\delta\left(i \lambda_{n}+\lambda_{0}\right)\left(r_{n}^{2}-\epsilon-1\right)}\right) \\
& \times\left[1+\prod_{m=1}^{N}\left(1-\delta\left(i \lambda_{m}+\lambda_{0}-\mu\right)\right)\right]^{2}, \quad\left(\Omega>\lambda_{0}>0\right)
\end{aligned}
$$

The path integral representation, and in particular the Trotter-Suzuki decomposition, only makes sense when $|\delta x| \ll 1$, where $x$ denotes anything that multiplies $\delta$ in the course of the calculation. Clearly, a finite $\left|\lambda_{n}\right| \leq \Omega$ is required in order to reexponentiate the fermionic contribution, or, in other words, to introduce a small parameter, allowing to neglect terms of order $\mathcal{O}\left(\delta^{2}\right)$, for our purpose. The square bracket in $Z_{\text {at }}$ therefore may be written as

$$
Z_{f}=\left[1+\prod_{n=1}^{N} e^{-\delta\left(i \lambda_{n}+\lambda_{0}-\mu\right)}\right]^{2}
$$


and we now obtain

$$
\begin{aligned}
& Z_{\text {at }}= \lim _{\epsilon \rightarrow 0^{+}} \lim _{\Omega \rightarrow \infty}\left(\prod_{n=1}^{N} \int_{-\Omega}^{\Omega} \frac{\delta d \lambda_{n}}{2 \pi} \int_{0}^{\infty} d\left(r_{n}^{2}\right)\right) \\
& {\left[e^{-\delta \sum_{n=1}^{N}\left(i \lambda_{n}+\lambda_{0}\right)\left(r_{n}^{2}-\epsilon-1\right)}+2 e^{\beta \mu} e^{-\delta \sum_{n=1}^{N}\left(i \lambda_{n}+\lambda_{0}\right)\left(r_{n}^{2}-\epsilon\right)}+e^{2 \beta \mu} e^{-\delta \sum_{n=1}^{N}\left(i \lambda_{n}+\lambda_{0}\right)\left(r_{n}^{2}-\epsilon+1\right)}\right] } \\
&=\lim _{\epsilon \rightarrow 0^{+}} \lim _{\Omega \rightarrow \infty}\left(\prod_{n=1}^{N} \int_{-\Omega}^{\Omega} \frac{\delta d \lambda_{n}}{2 \pi}\right) \\
& {\left[\prod_{n=1}^{N} \frac{e^{\delta\left(i \lambda_{n}+\lambda_{0}\right)(1+\epsilon)}}{\delta\left(i \lambda_{n}+\lambda_{0}\right)}+2 e^{\beta \mu} \prod_{n=1}^{N} \frac{e^{\delta\left(i \lambda_{n}+\lambda_{0}\right) \epsilon}}{\delta\left(i \lambda_{n}+\lambda_{0}\right)}+e^{2 \beta \mu} \prod_{n=1}^{N} \frac{e^{-\delta\left(i \lambda_{n}+\lambda_{0}\right)(1-\epsilon)}}{\delta\left(i \lambda_{n}+\lambda_{0}\right)}\right] . }
\end{aligned}
$$

The origin of $\epsilon$ is now clear: it prescribes how to close the contour for the second term in Eq. (14). A motivation, how to find $\epsilon$ and the bosonic Lagrange density in radial representation, is presented in Appendix A

In each term, the existence of the integrals is guaranteed by the shift of the $\lambda$-integration contour into the lower complex plane induced by a positive $\lambda_{0}$. The residue of the pole at $\lambda=i \lambda_{0}$ of the contour integration in the first two terms is finite and yields exactly the two contributions of $Z_{\mathrm{at}}$ : the hole line and the two particle lines with single occupancy of Fig. 1, respectively. The third term in Eq. (14), corresponding to double occupancy, is annihilated through the $\lambda$-integration, as expected from the implementation of the constraint.

It is instructive to invert the sequence of the integrations.

$$
\begin{aligned}
Z_{\mathrm{at}}= & \lim _{\epsilon \rightarrow 0^{+}} \prod_{n=1}^{N} \int_{0}^{\infty} d\left(r_{n}^{2}\right) \lim _{\Omega \rightarrow \infty} \int_{-\Omega}^{\Omega} \frac{\delta d \lambda_{n}}{2 \pi} \\
& {\left[e^{-\delta\left(i \lambda_{n}+\lambda_{0}\right)\left(r_{n}^{2}-\epsilon-1\right)}+2 e^{-\delta\left(i \lambda_{n}+\lambda_{0}\right)\left(r_{n}^{2}-\epsilon\right)} e^{\delta \mu}+e^{-\delta\left(i \lambda_{n}+\lambda_{0}\right)\left(r_{n}^{2}-\epsilon+1\right)} e^{2 \delta \mu}\right] } \\
= & \lim _{\epsilon \rightarrow 0^{+}} \prod_{n=1}^{N} \int_{0}^{\infty} d\left(r_{n}^{2}\right) \\
& {\left[e^{-\delta \lambda_{0}\left(r_{n}^{2}-1\right)} \hat{\delta}\left(r_{n}^{2}-1\right)+2 e^{-\delta \lambda_{0}\left(r_{n}^{2}-\epsilon\right)} \hat{\delta}\left(r_{n}^{2}-\epsilon\right) e^{\delta \mu}+e^{-\delta \lambda_{0}\left(r_{n}^{2}+1\right)} \hat{\delta}\left(r_{n}^{2}+1\right) e^{2 \delta \mu}\right] }
\end{aligned}
$$

where $\hat{\delta}$ is the Dirac $\delta$-function. The last line is equivalent to:

$$
Z_{\text {at }}=1+2 e^{\beta \mu} .
$$

In this way it appears that, after the projection has been performed in the first step, $r_{n}$ may only take two integer values, 0 or 1 in the last line of Eq. (15). We thus arrive at the conclusion that the integrations over the bosonic and the constraint fields can be replaced by the simple procedure demonstrated in Eq. (15).

To summarize, we have introduced a new path integral representation for slave bosons in the radial gauge. It is defined on a discretized time mesh, and has a well-defined continuum limit. Furthermore, we can take a more conventional approach (Eq. (10)), based on a coherent state functional integral for both fermions and bosons, or use an Ising-like variable representing the amplitude of empty sites (Eq. (15)). The extension to non-local terms in the Lagrangian will be discussed in Section IV where we also present a technique how to handle the integration over the amplitude field $r^{2}$.

\section{DISCRETE REPRESENTATION}

Besides the above integral representation with continuous fields, which may be used to set up various approximation schemes, one can alternatively obtain a discrete representation. Indeed the above calculation of the partition sum already suggests that it can be obtained without having to integrate over continuous values of $r_{n}$. Moreover integration over $\lambda_{n}$ mostly has the effect of "picking up" the appropriate value of $r_{n}$. This is achieved more elegantly by extending Coleman's projection scheme [31] to the case of a time-dependent constraint. For this purpose one introduces $\xi_{n} \equiv e^{-\delta\left(i \lambda_{n}+\lambda_{0}\right)}$, and rewrites the partition sum in the following compact form:

$$
Z_{\mathrm{at}}=\left.\sum_{\left\{r_{n}=0,1\right\}_{n}}\left(\prod_{n=1}^{N} \frac{\partial}{\partial \xi_{n}} \xi_{n}^{r_{n}}\right) \int \prod_{\sigma} D\left[f_{\sigma}, f_{\sigma}^{\dagger}\right] e^{-S_{f}}\right|_{\xi_{1}=\ldots=\xi_{N}=0}
$$


with

$$
S_{f}=\sum_{n=1}^{N} \sum_{\sigma} f_{n, \sigma}^{\dagger}\left[f_{n, \sigma}-f_{n-1, \sigma} \xi_{n} e^{\delta \mu}\right]
$$

In Eq. (16) and all following relations, $\sum_{\left\{r_{n}=0,1\right\}_{n}}$ denotes the "path integral" over the field $r$ which now is reduced to the set of discrete values $r_{n}=0,1$ for $n=1, \ldots, N$. We easily check that the atomic limit is reproduced:

$$
\begin{aligned}
Z_{\text {at }} & =\left.\sum_{\left\{r_{n}=0,1\right\}_{n}}\left(\prod_{n=1}^{N} \frac{\partial}{\partial \xi_{n}} \xi_{n}^{r_{n}}\right)\left(1+\prod_{m=1}^{N} \xi_{m} e^{\delta \mu}\right)^{2}\right|_{\xi_{1}=\ldots=\xi_{N}=0} \\
& =1+2 e^{\beta \mu} .
\end{aligned}
$$

This discretized representation is certainly the most direct way to construct the partition sum - and later on the Green's function - as a sum of free fermionic paths, controlled by a set of Ising variables $r_{1}$ to $r_{N}$.

\section{A. Representation of the Grassmann fields for a physical electron.}

In the radial gauge we have the following assignment of electron fields to the previously introduced auxiliary fields:

$$
\begin{aligned}
& c_{n, \sigma}^{\dagger}=r_{n} f_{n, \sigma}^{\dagger} \\
& c_{n, \sigma}=r_{n+1} f_{n, \sigma}
\end{aligned}
$$

The product composition is just the expression Eq. (1) - only the choice of the time steps has to be confirmed. This is easily derived from the requirement that in order to attain a non-zero result for the Green's function we need "complete chains" in the Grassmann integration. We first clarify the term "complete chain" in the following paragraph, and then convince ourselves in Subsection IIIB that the choice of time steps in Eq. (19) is necessary to obtain a finite electron propagator.

"Complete chain" denotes a product of Grassmann numbers over all time steps, either of the form $\prod_{n=1}^{N} f_{n, \sigma} f_{n, \sigma}^{\dagger}$ or, equivalently, as $\prod_{n=1}^{N} f_{n, \sigma}^{\dagger} f_{n-1, \sigma}$ which both result from the expansion of the exponentiated action, and only the integration of such complete chains results in a non-zero value of the path integral. The first form can be characterized as a "hole chain" since it corresponds to the hole line in Fig. 1 (top line). Actually, this hole contribution to $Z_{\text {at }}$ with value 1 is formed by the product of two hole chains $\left(\prod_{n=1}^{N} f_{n, \uparrow} f_{n, \uparrow}^{\dagger}\right)\left(\prod_{n=1}^{N} f_{n, \downarrow} f_{n, \downarrow}^{\dagger}\right)$. In the presented slave boson scheme, this double hole chain is matched by a complete chain of $r_{n}$ variables with value 1 (the first contribution in the last line of Eq. (15)). The second arrangement $\prod_{n=1}^{N} f_{n, \sigma}^{\dagger} f_{n-1, \sigma}$ then should be interpreted as a "particle chain" which carries spin $\sigma$. Indeed, the dashed lines with value $\exp (\mu \beta)$ in Fig. 1 are formed by $\left(\prod_{n=1}^{N} f_{n,-\sigma} f_{n,-\sigma}^{\dagger}\right)\left(\prod_{n=1}^{N} f_{n, \sigma}^{\dagger} f_{n-1, \sigma} e^{\delta \mu}\right)$ with $\sigma=\uparrow, \downarrow$ for the two lines, respectively. Finally, the third contribution in the last line of Eq. (15) is generated from a double particle line $\left(\prod_{n=1}^{N} f_{n,-\sigma}^{\dagger} f_{n-1,-\sigma} e^{\delta \mu}\right)\left(\prod_{n=1}^{N} f_{n, \sigma}^{\dagger} f_{n-1, \sigma} e^{\delta \mu}\right)$ but it is projected out via $\hat{\delta}\left(r_{n}^{2}+1\right)$, as we work in the constrained Fock space with no double occupancies.

\section{B. Calculation of the Green's function.}

For the Green's function $G_{\sigma}\left(\tau_{f}-\tau_{i}\right)$ — with electron creation at time step $\tau_{i}$ and annihilation at $\tau_{f}$ - the complete chains again have to exist. However they are built from a distinct arrangement of Grassmann variables from the Lagrangian and the physical electron creation and annihilation operators. In this case, the hole chain runs only from time step 0 to the time step when the $\sigma$-electron is created (visualized in Fig. 2, where the electron creation is at time step 1). The $\sigma$-hole chain is then replaced by a $\sigma$-particle chain in between time steps 1 and $m$. This product of hole- and particle chains still results in a finite value for the Grassmann integrations since the electron creation and annihilation operators at time steps 1 and $m$ provide the missing Grassmann numbers in order to produce a "complete chain" from 0 to $N$.

In order to verify this arrangement and to understand the shift of the time arguments by one time step in the relation for $c_{n, \sigma}$ in Eq. 19), we explicitly carry out the evaluation of the Green's function

$$
-Z G_{\sigma}(m-1) \equiv\left\langle c_{m, \sigma} c_{1, \sigma}^{\dagger}\right\rangle=\left\langle r_{m+1} f_{m, \sigma} r_{1} f_{1, \sigma}^{\dagger}\right\rangle
$$


in the atomic limit. Obviously the $\sigma$-fermion exists for $m-1$ time steps. When we integrate out the Grassmann fields as above for $Z_{\text {at }}$ we have:

$$
-Z_{\mathrm{at}} G_{\mathrm{at}, \sigma}(m-1)=\left.\sum_{\left\{r_{n}=0,1\right\}_{n}}\left(\prod_{n=1}^{N} \frac{\partial}{\partial \xi_{n}} \xi_{n}^{r_{n}}\right) r_{m+1} r_{1}\left(1+\prod_{m=1}^{N} \xi_{m} e^{\delta \mu}\right)^{2} D_{m, 1}^{-1}\right|_{\xi_{1}=\ldots=\xi_{N}=0}
$$

Here the integration over the $-\sigma$-fermion produces one factor $\left(1+\prod_{m=1}^{N} \xi_{m} e^{\delta \mu}\right) \equiv \operatorname{det}[D]$, and the integration over the $\sigma$-fermion yields the propagator matrix-element $D_{m, 1}^{-1}$ multiplied by the second factor det $[D]$. This propagator is:

$$
D_{m, 1}^{-1} \equiv \prod_{i=2}^{m}\left(\xi_{i} e^{\delta \mu}\right) / \operatorname{det}[D]
$$

Next we calculate the derivatives at each time step and obtain

$$
-Z_{\mathrm{at}} G_{\mathrm{at}, \sigma}(m-1)=\sum_{\left\{r_{n}=0,1\right\}_{n}} r_{m+1} r_{1}\left(\delta_{r_{1}, 1} \delta_{r_{2}, 0} \ldots \delta_{r_{m}, 0} \delta_{r_{m+1}, 1} \ldots \delta_{r_{N}, 1}\right) e^{\delta \mu(m-1)}
$$

As anticipated above, we produced a chain of $r$-values, with $r_{i}=0$ for $2 \leq i \leq m$. We find the correct expression for this projected atomic Green's function

$$
-Z_{\mathrm{at}} G_{\mathrm{at}, \sigma}(m-1)=e^{\mu(m-1) \delta} .
$$

If we had erroneously taken $c_{n, \sigma}=r_{n} f_{n, \sigma}$ in Eqs. (19) and (20), we would have to replace $r_{m+1}$ by $r_{m}$ in Eq. (23), and the result would be incorrectly zero, due to the $\delta_{r_{m}, 0}$ in the $r$-chain. Higher order correlation functions can be calculated in a similar way.

We summarize that the expressions for the decomposition of the $c$-operators (Eq. (19)), together with the expression of the local part of the action (Eq. (11)) and the measure of Eq. (10) form a complete representation of the slave boson functional integral in the radial gauge in the limit of infinitely strong local interaction. In the following chapter we will discuss non-local terms in the Lagrangian, using exactly this representation.

\section{NON-LOCAL LAGRANGIAN}

Two types of non-local terms have to be included for a full discussion of physical models for correlated electrons: kinetic terms, i.e. hopping of electrons, and non-local interactions.

We first consider kinetic terms of the type (with $n_{i, \sigma}=c_{i, \sigma}^{\dagger} c_{i, \sigma}$ ):

$$
H_{t}=\sum_{i, j, \sigma} t_{i, j}\left(1-n_{i,-\sigma}\right) c_{i, \sigma}^{\dagger} c_{j, \sigma}\left(1-n_{j,-\sigma}\right)
$$

which constrain the hopping of electrons (with hopping amplitude $t_{i, j}$ ) to the low energy sector of the Fock space, i.e. doubly occupied sites are not created. In the language of radial-gauge slave bosons we have

$$
S_{t}=\sum_{n} \sum_{i, j, \sigma} \delta t_{i, j} r_{i, n+1} f_{i, n+1, \sigma}^{\dagger} f_{j, n, \sigma} r_{j, n+1}
$$

using the relations Eq. (19). A formal solution of a lattice model with arbitrary hopping is possible since the action is bilinear in $f_{\sigma}^{(\dagger)}$ and it is found in analogy to the atomic limit of the previous section. The $\xi$-derivatives, which enforce the constraint, are now taken at each time step and each lattice site:

$$
Z=\left.\sum_{\left\{r_{i, n}=0,1\right\}_{i, n}}\left(\prod_{i, n} \frac{\partial}{\partial \xi_{i, n}} \xi_{i, n}^{r_{i, n}}\right) \operatorname{det}\left[D\left[\xi_{i, n}\right]\right]^{2}\right|_{\xi_{i, 1}=\ldots=\xi_{i, N}=0}
$$

with the fermionic inverse propagator matrix

$$
D_{i j, n n^{\prime}}\left[\xi_{i, n}\right]=\hat{\delta}_{i, j} D_{i, n n^{\prime}}^{(0)}+\delta t_{i, j} r_{j, n+1} r_{i, n+1} \hat{\delta}_{n, n^{\prime}+1}
$$


and its local part:

$$
D_{i, n n^{\prime}}^{(0)}=\hat{\delta}_{n, n^{\prime}}-\xi_{i, n} e^{\delta \mu} \hat{\delta}_{n, n^{\prime}+1}
$$

It is understood that the anti-periodicity is taken care of implicitly. Although the sum over $\left\{r_{i, n}=0,1\right\}_{i, n}$ runs over all possible values of "hole occupancies" on each site and at each time step, "unphysical paths" will be projected out due to the product of $\xi$-derivatives. Hereby, unphysical paths are the disconnected paths of the variable $r_{i, n}$ [32]. This observation simplifies the calculation considerably. An explicit example is presented in Appendix B, where the partition sum of the two-site cluster is calculated.

Secondly we consider non-local interactions. The infinite- $U$ Hubbard model, that we considered so far, is a highly idealized model which is unlikely to be realized in nature. In the attempt to set up a lattice model for interacting electrons, one has to realize that many other non-local interaction terms may contribute, (for a recent discussion see Vollhardt et al. [33]). On top of the expected density-density interactions, we also encounter spin-exchange terms, correlated hopping terms, four-site terms, etc.. Here, using our representation, it turns out that the density-density interaction term, $S_{\text {nloc }}=\sum_{n} \sum_{i, j, \sigma, \sigma^{\prime}} \delta V_{i, j} n_{i, n, \sigma} n_{j, n, \sigma^{\prime}}$, can be included without introducing a fermionic interaction term. Rather, the contribution to the action may be rewritten in the form:

$$
S_{\mathrm{nloc}}=\sum_{n} \sum_{i, j} \delta V_{i, j}\left(1-r_{i, n}\right)\left(1-r_{j, n}\right)
$$

where we used the identity $r_{i, n}^{2}=r_{i, n}$, valid in the physical subspace. This Gaussian form of the "interaction term" has to be contrasted with the canonical fermionic representation and the Cartesian representation for slave bosons where the action remains a true interaction term. In both latter cases, it cannot be treated in a straightforward fashion. However, here it appears as additional Boltzmann factors in the expansion of the partition sum Eq. (27).

The last detail that prevents us from taking advantage of both the radial representation and a conventional propagator expansion is the integration range for the bosonic field. Indeed it would be very tempting to extend it from $[0, \infty]$ to $[-\infty, \infty]$, such that one could work in momentum space. However such a step is unlikely to yield a meaningful answer, since double occupancy is related to the value $r_{n}^{2}=-1$ (see Eq. (15)), which would then be inside the integration range. This difficulty may nevertheless be circumvented by i) introducing a new integration variable $x_{n} \equiv r_{n}^{2}$, ii) making use of the fact that $r_{n}^{2}=r_{n}$ in the physical subspace, iii) adding a potential term $W x_{n}\left(x_{n}-1\right)$ that precisely vanishes in the physical subspace, iv) extending the integration range for $x_{n}$ to $[-\infty, \infty]$ and, v) sending $W$ to infinity at the end of the calculation that would definitely project out any occupancy different from zero and one. This transforms the bosonic part of the action $S_{b}$ in Eq. (11) into:

$$
S_{b}=\delta \sum_{n}\left(\left(i \lambda_{n}+\lambda_{0}\right)\left(x_{n}-1\right)+W x_{n}\left(x_{n}-1\right)\right) .
$$

We skipped $\epsilon$ here and in the following equations since $x_{n}$ now extends to negative values. Integration over $x_{n}$ with measure one, now from $-\infty$ to $+\infty$, yields, in combination with Eq. (13), the partition sum in the atomic limit:

$$
\begin{aligned}
Z_{\text {at }} & =\lim _{W \rightarrow \infty}\left(\prod_{n=1}^{N} \int_{-\infty}^{\infty} \frac{\delta d \lambda_{n}}{2 \pi} \sqrt{\frac{\pi}{\delta W}} e^{\delta\left(i \lambda_{n}+\lambda_{0}\right)} e^{\delta W\left(\frac{i \lambda_{n}+\lambda_{0}-W}{2 W}\right)^{2}}\right)\left[1+\prod_{m=1}^{N} e^{-\delta\left(i \lambda_{m}+\lambda_{0}-\mu\right)}\right]^{2} \\
& =\lim _{W \rightarrow \infty}\left(1+2 e^{\beta \mu}+e^{2 \beta(\mu-W)}\right)
\end{aligned}
$$

As anticipated above double-occupancy is annihilated by taking the limit $W \rightarrow \infty$ while, in the opposite limit $W \rightarrow 0$, i.e. hadn't we included this potential term as is customary in slave boson calculations [23], the third contribution could well carry a substantial weight 34 . This scheme can be extended to the calculation of the Green's function, and in contrast to the above calculation (Eq. (32)), $W$ does not enter the result for $Z_{\mathrm{at}} G_{\mathrm{at}, \sigma}(\tau)$, as explained in Appendix D. Consequently, in a hopping expansion of the partition sum, $W$ would be related only to those paths where there is no change in occupancy on one or several sites over the entire imaginary time interval.

Finally we apply this scheme to lattice models. The measure of the functional integral is the same as for the single site problem except that all fields now carry a site index. The potential term, introduced above to enforce physical values for the amplitude of the slave bosons, may be implemented as a global term. It is independent of the site index. In summary, we find for spin- $\frac{1}{2}$ fermions on a lattice, interacting through an arbitrary Coulomb-like interaction, which is locally infinitely strong, the following expressions for the action in the language of radial-gauge slave bosons:

$$
S=S_{f}+S_{b}+S_{t}
$$


where:

$$
\begin{aligned}
S_{f} & =\sum_{i, n, \sigma} f_{i, n, \sigma}^{\dagger}\left[f_{i, n, \sigma}-f_{i, n-1, \sigma} e^{-\delta\left(i \lambda_{i, n}-\mu\right)}\right] \\
S_{b} & =\delta \sum_{i, n}\left(i \lambda_{i, n}\left(x_{i, n}-1\right)+W x_{i, n}\left(x_{i, n}-1\right)+V_{i, j}\left(1-x_{i, n}\right)\left(1-x_{j, n}\right)\right) \\
S_{t} & =\sum_{n} \sum_{i, j, \sigma} \delta t_{i, j} x_{i, n+1} f_{i, n+1, \sigma}^{\dagger} f_{j, n, \sigma} x_{j, n+1}
\end{aligned}
$$

and the partition sum is given by:

$$
Z=\lim _{W \rightarrow \infty}\left(\prod_{i, n} \int \prod_{\sigma} D\left[f_{i, n, \sigma}, f_{i, n, \sigma}^{\dagger}\right] \int_{-\infty}^{\infty} \frac{\delta d \lambda_{i, n}}{2 \pi} \int_{-\infty}^{\infty} d x_{i, n}\right) e^{-S}
$$

The measure is now trivial, and the interaction terms included in $S_{b}$ are bilinear. As an example of how this method works for a lattice problem we solve the Ising chain in Appendix $\mathrm{C}$.

\section{CONCLUSIONS}

Models of strongly interacting lattice fermions have been the focus of many publications on the Mott-Hubbard metal-insulator transition, itinerant magnetism and strange metallic phases in recent years. Apart from the thorough analysis of the Anderson impurity case [25], slave boson approaches have been mainly restricted to saddle point evaluations. However low energy spin fluctuations, for example, cannot be implemented adequately, as quantum spin fluctuations are only included as perturbation. Furthermore, this technique is not suitable to discuss non-Fermi liquid behavior, as corrections of higher order in $1 / \mathcal{N}$ have to be included.

This necessitates to further pursue non-perturbative slave boson approaches. Here we considered the construction of a functional-integral formalism in the radial gauge which is not restricted to fluctuations around the $\mathcal{N}=\infty$ saddle point. It is defined on a discretized time mesh, and has a well-defined continuum limit. We restricted our considerations to models in the limit of large (positive) on-site energy, that is, we work in the constrained Fock space with no double occupancy.

These models allow, in the radial gauge, to perform the functional integrals with real bosonic fields. The phase(s) can be absorbed in the constraint Lagrange multipliers which are thereby promoted to real (time-dependent) fields. Dynamics of the radial fields themselves does not exist (as discussed in Appendix A). They serve to enforce the constraint for each time step by keeping track of the motion of empty sites, as exemplified for the local problem in Eqs. (15) and (23), and for the itinerant problem in Eq. (28). This procedure can be extended to other slave boson representations as, for example, to the Kotliar-Ruckenstein slave bosons, the representation of which is given for the Ising chain in Appendix 9 [35.

Non-local terms in general render the functional integral unsolvable. Yet Coulomb-like terms with non-local densitydensity interactions can be rewritten in bilinear form with radial fields, that is, they represent Gaussian terms in the functional integral (Eq. (30) in Section IV, and also other non-local interactions may be rewritten in bilinear form, e.g., the Ising spin coupling within the Kotliar-Ruckenstein representation, see Appendix (Q). This is neither possible in the canonical fermionic representation nor in the Cartesian representation for slave bosons. These bilinear terms in the Lagrangian generate a finite dispersion for the (radial) slave particles. In order to keep the bosonic part Gaussian and to allow for a momentum space representation, one has to extend the range of integration for the square of the radial field to minus infinity. It can be implemented with the observation that the functional integration stays correct if we add an additional global Gaussian term, that is, a potential term $W\left(x_{i, n}-1\right) x_{i, n}$ where $x_{i, n}$ is the square of the radial part of the slave boson, and send $W \rightarrow \infty$ at the end. This proved to be a valid procedure to constrain the amplitude of the slave boson to the physical values 0 and 1, without introducing any additional complications (see Appendices $\mathrm{G}$ and D, and the paragraph with Eqs. (31) and (32) in Section [V).

In this article we advanced two mechanisms for the solution of a functional integral for constrained electronic problems: either we keep continuous fields which are originally introduced on a discrete time mesh but have a welldefined continuum limit, or we enforce the constraint in the first step and thereby reduce the slave boson radial field to an Ising-like variable in a discrete representation. The first approach is summarized in the final expressions Eqs. (34) and (35) - and for Kotliar-Ruckenstein slave bosons in Eq. (C2) - in which the bosonic fields are real fields and the bosonic action is of bilinear form. The second approach is discussed in Section III, and extended to itinerant 
problems in Section IV, Eqs. (27) and (29), which can be taken as a starting point for a numerical evaluation in the strong coupling limit that does not build on a Hirsch decoupling. Furthermore, non-local interactions can be included without the need of any additional decoupling.

\section{ACKNOWLEDGMENTS}

We gratefully thank M. Dzierzawa and P. Wölfle for several stimulating discussions, and H. Beck and J.-P. Derendinger for interesting discussions. R. F. is grateful for the warm hospitality at the Institut für Theorie der Kondensierten Materie of Karlsruhe University, and the EKM of Augsburg University where part of this work has been done. T. K. greatly enjoyed the hospitality at the Université de Neuchâtel. We acknowledge the financial support by the fonds national suisse de la recherche scientifique, the BMBF 13N6918/1 and Sonderforschungsbereich 484 of the Deutsche Forschungsgemeinschaft.

\section{APPENDIX A:}

Below we motivate why the radial amplitude field $r_{n}$ has no dynamics and, furthermore, why the limit of integration is shifted to the negative real axis by an infinitesimal amount $\epsilon$. We start from $S_{b}$ Eq. (5) in Cartesian representation.

$$
S_{b}=\sum_{n}\left[b_{n}^{*}\left(b_{n}-b_{n-1}\right)+i \lambda \delta b_{n}^{*} b_{n-1}\right]
$$

and substitute $b_{n}=r_{n} e^{i \varphi_{n}}$.

$$
\begin{aligned}
S_{b} & =\sum_{n}\left[r_{n}\left(r_{n}-r_{n-1}\right)+r_{n} r_{n-1}\left(1-e^{i \delta \frac{\varphi_{n-1}-\varphi_{n}}{\delta}}(1-i \delta \lambda)\right)\right] \\
& =\sum_{n} i \delta\left(\dot{\varphi}_{n}+\lambda\right)\left[r_{n}^{2}-\delta r_{n} \dot{r}_{n}\right]+\delta r_{n} \dot{r}_{n}+\text { "further terms in } \mathcal{O}\left(\delta^{2}\right)^{\prime \prime}+\mathcal{O}\left(\delta^{3}\right)
\end{aligned}
$$

In the last line we observe that $\sum_{n} \delta r_{n} \dot{r}_{n} \rightarrow \frac{\beta}{2} \int_{0}^{\beta} d \tau \frac{d r^{2}}{d \tau}=0$ (due to periodic boundary conditions for the bosonic field). The "further terms in $\mathcal{O}\left(\delta^{2}\right)$ " can all be neglected with respect to the linear terms. Here we introduced the time-dependent constraint field $\lambda_{n} \equiv \dot{\varphi}_{n}+\lambda$. The included term quadratic in $\delta$ is special: if $-\delta r_{n} \dot{r}_{n}$ is positive, it is always a negligible correction to $r_{n}^{2}$. However, for $\delta r_{n} \dot{r}_{n} \geq 0$ it may interpreted as an infinitesimal shift $\epsilon$ of $r_{n}^{2}$ to negative values for $r_{n} \rightarrow 0$. Consequently, the leading term in $S_{b}$ now reads:

$$
S_{b}=\sum_{n} i \delta \lambda_{n}\left(r_{n}^{2}-\epsilon\right)
$$

There is no dynamic term of the field $r_{n}$ - as there is one for $b_{n}$ in Eq. (A1) - since this amplitude field is a real field. On account of the constraint we avoid the essential difficulty that one encounters when one uses polar coordinates as discussed by Edwards and Gulyaev [30]. Particularly, the part of the action in polar coordinates, that corresponds to the Gaussian part of the action in Cartesian coordinates, cannot be written in a Gaussian form. This is true even to first order in $\delta$, and in 30 higher order terms in the polar fields must be kept as well.

\section{APPENDIX B:}

As an illustration of how our discrete representation yields the partition sum we explicitly calculate it for the twosite cluster. An instructive "warm up" exercise consists in discretizing the imaginary time axis into six time steps. In order to evaluate $Z$ Eq. (27) we first choose the physical path:

$$
\begin{aligned}
& r_{1}=r_{2}=1 ; \quad r_{3}=r_{4}=0 ; \quad r_{5}=r_{6}=1 \quad \text { on site } 1 \\
& \tilde{r}_{1}=0 ; \quad \tilde{r}_{2}=\ldots=\tilde{r}_{5}=1 ; \quad \tilde{r}_{6}=0 \quad \text { on site } 2
\end{aligned}
$$


Here and in the following we use $\tilde{x}_{n} \equiv x_{i=2, n}$ for anything on site 2 . To built up the inverse propagator matrix we observe that all $\xi$ 's for which $r$ is equal to 1 can be set to zero, since the derivatives with respect to those $\xi$ 's have to be taken from the prefactor. As a result the inverse propagator matrix is:

$$
D=\left(\begin{array}{cccccccccccc}
1 & 0 & 0 & 0 & 0 & 0 & 0 & 0 & 0 & 0 & 0 & 0 \\
0 & 1 & 0 & 0 & 0 & 0 & -\delta t & 0 & 0 & 0 & 0 & 0 \\
0 & -\xi_{3} e^{\delta \mu} & 1 & 0 & 0 & 0 & 0 & 0 & 0 & 0 & 0 & 0 \\
0 & 0 & -\xi_{4} e^{\delta \mu} & 1 & 0 & 0 & 0 & 0 & 0 & 0 & 0 & 0 \\
0 & 0 & 0 & 0 & 1 & 0 & 0 & 0 & 0 & -\delta t & 0 & 0 \\
0 & 0 & 0 & 0 & 0 & 1 & 0 & 0 & 0 & 0 & 0 & 0 \\
0 & 0 & 0 & 0 & 0 & 0 & 1 & 0 & 0 & 0 & 0 & \tilde{\xi}_{1} e^{\delta \mu} \\
-\delta t & 0 & 0 & 0 & 0 & 0 & 0 & 1 & 0 & 0 & 0 & 0 \\
0 & 0 & 0 & 0 & 0 & 0 & 0 & 0 & 1 & 0 & 0 & 0 \\
0 & 0 & 0 & 0 & 0 & 0 & 0 & 0 & 0 & 1 & 0 & 0 \\
0 & 0 & 0 & -\delta t & 0 & 0 & 0 & 0 & 0 & 0 & 1 & 0 \\
0 & 0 & 0 & 0 & 0 & 0 & 0 & 0 & 0 & 0 & -\tilde{\xi}_{6} e^{\delta \mu} & 1
\end{array}\right)
$$

Along this path the hole hops from site 1 to site 2 at time 2, and back to site 1 at time 5 . Accordingly one would expect that $t$ only enters $D$ on two entries: $(2, \tilde{1})$ and $(\tilde{4}, 5)$. But it also appears at two other entries. Nevertheless the latter two entries, which do not correspond to physical processes, are easily seen not to contribute to the determinant. The latter reads:

$$
\operatorname{det}[D]=1+(\delta t)^{2} e^{4 \delta \mu} \xi_{3} \xi_{4} \tilde{\xi}_{1} \tilde{\xi}_{6}
$$

If we now let the number of time steps to be $N$ and gather all second order processes in $t$ (their number being $N(N-1))$, we obtain their contribution to the partition sum as

$$
Z_{2}=2(\beta t)^{2} e^{(N-2) \delta \mu}
$$

as it should. With some patience one may extend this procedure to an arbitrary number of hopping processes, and we checked that they can all be summed up to

$$
Z=1+2 e^{\beta \mu} 2 \cosh (\beta t)
$$

which is the correct answer.

\section{APPENDIX C:}

In this appendix we illustrate the method described in Section IV for the Ising chain. To that aim we make use of the Kotliar and Ruckenstein representation of the Hubbard model $[8]$ in the limit $U \rightarrow \infty$. We thus introduce two auxiliary fermions $f_{\uparrow}$ and $f_{\downarrow}$, and three auxiliary bosons $e$, representing empty sites, and $p_{\uparrow}$ and $p_{\downarrow}$ representing singly occupied sites. On each site $i$ they are subject to three constraints:

$$
\begin{gathered}
e_{i}^{\dagger} e_{i}+\sum_{\sigma} p_{i, \sigma}^{\dagger} p_{i, \sigma}=1 \\
p_{i, \sigma}^{\dagger} p_{i, \sigma}=f_{i, \sigma}^{\dagger} f_{i, \sigma} \quad \sigma=\uparrow, \downarrow
\end{gathered}
$$

which are respectively enforced by three Lagrange multipliers denoted by $\alpha_{i}$ and $\lambda_{i, \sigma}$. The phase of all three bosons can be gauged away [9, 36, 37, and the three Lagrange multipliers are promoted to fields. Since, for the Ising model, we are working at exactly one electron per site, the $e$-field, representing empty sites, has to be fixed to zero. We also could implement this "half-filling condition" in the standard way by introducing a chemical potential for the electrons. However here, it is more convenient to introduce a Kronecker $\delta$-function $\hat{\delta}_{e_{i, n}, 0}^{K}$ in the measure (see Eq. (C3) below). Just setting the $e$-field to zero from the outset would introduce a spurious divergence in the measure. The action is thus: 


$$
\begin{aligned}
S & =\sum_{i, n, \sigma} f_{i, n, \sigma}^{\dagger}\left[f_{i, n, \sigma}-f_{i, n-1, \sigma} e^{-i \delta \lambda_{i, n, \sigma}}\right] \\
& +\delta \sum_{i, n, \sigma}\left(W p_{i, n, \sigma}\left(p_{i, n, \sigma}-1\right)-i \lambda_{i, n, \sigma} p_{i, n, \sigma}\right)+\delta \sum_{i, n} \alpha_{i, n}\left(\sum_{\sigma} p_{i, n, \sigma}+e_{i, n}-1\right) \\
& +\delta \sum_{i, j, n} J_{i, j}\left(p_{i, n, \uparrow}-p_{i, n, \downarrow}\right)\left(p_{j, n, \uparrow}-p_{j, n, \downarrow}\right) .
\end{aligned}
$$

It consists of a fermionic part, the first contribution, and the remaining ones form the bosonic part $S_{b}$. Here, $J_{i, j}$ is the Ising-spin coupling, and $W$ was introduced in Eq. (31) and the preceding discussion. After the integration over the fermions we obtain the partition sum as:

$$
\begin{aligned}
Z & =\lim _{W \rightarrow \infty}\left\{\prod_{i}\left[\prod_{n=1}^{N} \int_{-\infty}^{\infty} \frac{\delta d \alpha_{i, n}}{2 \pi} \int_{-\infty}^{\infty} \prod_{\sigma} \frac{\delta d \lambda_{i, n, \sigma}}{2 \pi} \int_{-\infty}^{\infty} \prod_{\sigma} d p_{i, n, \sigma} \int_{-\infty}^{\infty} d e_{i, n} \hat{\delta}_{e_{i, n}, 0}^{K}\right]\right\} \\
& \times\left[\prod_{i, \sigma}\left(1+\prod_{n=1}^{N} e^{-i \delta \lambda_{i, n, \sigma}}\right)\right] e^{-S_{b}} .
\end{aligned}
$$

We first integrate over the constraint fields $\lambda_{\sigma}$, then over $\alpha$ and finally over $e$. As a result the term with $W$ drops out since the $\lambda_{\sigma}$-integrations already enforce the constraint to the point that $p_{i, n, \sigma}$ can take only the discrete values 0 and 1 . Furthermore we observe that $p_{i, n, \sigma}$ loses its time-dependence since the previous integrations resulted in the two straight "world lines" $\prod_{n} \hat{\delta}\left(p_{i n \uparrow}\right) \hat{\delta}\left(p_{i n \downarrow}-1\right)+\prod_{n} \hat{\delta}\left(p_{i n \uparrow}-1\right) \hat{\delta}\left(p_{i n \downarrow}\right)$ for each site $i$. Thus, setting $p_{i, \sigma} \equiv p_{i, 1, \sigma}=\ldots=p_{i, N, \sigma}$ we get:

$$
\begin{aligned}
Z & =\left[\prod_{i} \int_{-\infty}^{\infty} d p_{i, \uparrow} \int_{-\infty}^{\infty} d p_{i, \downarrow}\left(\hat{\delta}\left(p_{i, \uparrow}\right) \hat{\delta}\left(p_{i, \downarrow}-1\right)+\hat{\delta}\left(p_{i, \uparrow}-1\right) \hat{\delta}\left(p_{i, \downarrow}\right)\right)\right] \\
& \times e^{-\beta \sum_{i, j} J_{i, j}\left(p_{i, \uparrow}-p_{i, \downarrow}\right)\left(p_{j, \uparrow}-p_{j, \downarrow}\right)}
\end{aligned}
$$

which holds for any coupling matrix $J_{i, j}$ and topology. If we now restrict ourselves to nearest neighbor interaction and a chain of length $L$ with open boundary conditions, we can recursively integrate over $p_{1, \uparrow}, p_{1, \downarrow}, \ldots, p_{L, \uparrow}$, and $p_{L, \downarrow}$ to obtain the known result

$$
Z=2(2 \cosh (\beta J))^{L-1}
$$

\section{APPENDIX D:}

We calculate the Green's function in the atomic limit using the action Eq. (34) in which a global constraint was introduced so that the radial amplitudes can be integrated from $-\infty$ to $\infty$. In combination with the expression of the Green's function Eq. (20) and the propagator Eq. (22) we get:

$$
\begin{aligned}
Z_{\text {at }} G_{\text {at }, \sigma}(m-1) & =\lim _{W \rightarrow \infty}\left(\prod_{n=1}^{N} \int_{-\infty}^{\infty} d x_{n} \int_{-\infty}^{\infty} \frac{\delta d \lambda_{n}}{2 \pi} e^{-\delta\left(i \lambda_{n}\left(x_{n}-1\right)+W x_{n}\left(x_{n}-1\right)\right)}\right) x_{1} x_{m+1} \\
& \times\left(1+e^{-\delta \sum_{i=1}^{N}\left(i \lambda_{i}-\mu\right)}\right) e^{-\delta \sum_{i=2}^{m}\left(i \lambda_{i}-\mu\right)}
\end{aligned}
$$

This relation is easily verified either by straightforward integration over the Grassmann fields or, more directly, by realizing that in Eq. (21) one has to replace $\xi_{n} \rightarrow e^{-i \delta \lambda_{n}}$ in the propagator $D^{-1}$ and in $\operatorname{det} D$, and $\left(\partial \xi_{n}^{r_{n}} / \partial \xi_{n}\right)$ is replaced by the bosonic path integral $\int_{-\infty}^{\infty} \frac{\delta d \lambda_{n}}{2 \pi} e^{-\delta\left(i \lambda_{n}\left(x_{n}-1\right)+W x_{n}\left(x_{n}-1\right)\right)}$. Integration over $\lambda$ yields:

$$
\begin{aligned}
& Z_{\mathrm{at}} G_{\mathrm{at}, \sigma}(m-1)=\lim _{W \rightarrow \infty} e^{\delta \mu(m-1)}\left(\prod_{n=1}^{N} \int_{-\infty}^{\infty} d x_{n} e^{-\delta W x_{n}\left(x_{n}-1\right)}\right) x_{1} x_{m+1} \\
\times & {\left[\hat{\delta}\left(x_{1}-1\right)\left(\prod_{n=2}^{m} \hat{\delta}\left(x_{n}\right)\right)\left(\prod_{n=m+1}^{N} \hat{\delta}\left(x_{n}-1\right)\right)+e^{\beta \mu} \hat{\delta}\left(x_{1}\right)\left(\prod_{n=2}^{m} \hat{\delta}\left(x_{n}+1\right)\right)\left(\prod_{n=m+1}^{N} \hat{\delta}\left(x_{n}\right)\right)\right] } \\
= & e^{\delta \mu(m-1)}
\end{aligned}
$$


The second contribution above, which is related to double occupancy, cancels due to the additional projection in the external electron operators, represented by the factors $x_{1} x_{m+1}$ in Eq. (20). For this reason $W$ drops out of the calculation of the Green's function, in contrast to the calculation of the partition sum. This holds for the calculation of higher correlation functions as well. 


\section{Figure captions}

Figure 1: Contributions to the atomic partition sum for infinite on-site interaction $U$.

Figure 2: Time evolution of the fields $r$ and $f_{\sigma}$ in the imaginary-time local Green's function, $G_{\sigma}(m-1)$. The dashed line is a "particle chain" (see text for details). "Hole chains" are not depicted. 
a Permanent address: Laboratoire Crismat-ISMRA, 6, Bld. du Maréchal Juin, 14050 Caen Cedex 4, France.

[1] Y. Vilk, and A.-M. S. Tremblay, J. Phys. I France 7, 1309 (1997).

[2] P. W. Anderson, The Theory of Superconductivity in the High- $T_{c}$ Cuprates (Princeton University Press, Princeton, 1997).

[3] S. Pairault, D. Sénéchal, and A.-M. S. Tremblay, Phys. Rev. Lett. 80, 5389 (1998), and condmat/9905242.

[4] Ph. Brune and A. P. Kampf, condmat/0001210.

[5] In a different approach to these effective models, a degenerate perturbation theory is set up ab initio so that a Hamilton operator in the projected Fock space, restricted to low-energy excitations, can be determined: M. Takahashi, J. Phys. C 10, 1289 (1977).

[6] E. O. Tüngler, and T. Kopp, Nucl. Phys. B 443, 516 (1995).

[7] S. E. Barnes, J. Phys. F 6, 1375 (1976); F 7, 2631 (1977).

[8] G. Kotliar, and A. E. Ruckenstein, Phys. Rev. Lett. 57, 1362 (1986).

[9] R. Frésard, and P. Wölfle, Proceedings of the Adriatico Research Conference and Miniworkshop "Strongly Correlated Electron Systems III", Eds. G. Baskaran, A. E. Ruckenstein, E. Tosatti et Yu Lu, Int. J. of Mod. Phys. B 6, 685 (1992).

[10] However Bose condensation may be suppressed, even on a mean field level, and bond pairing of bosons emerges instead if the constraint is recast into an interaction term between the various slave particles: T. Kopp, F. J. Seco, S. Schiller, and P. Wölfle, Phys. Rev. B 38, 11835 (1988).

[11] W. Metzner, and D. Vollhardt, Phys. Rev. Lett. 62, 324 (1989); W. Metzner, Z. Phys. B 77253 (1989); W. Metzner, and D. Vollhardt, Phys. Rev. B 37, 7382 (1988).

[12] L. Lilly, A. Muramatsu, and W. Hanke, Phys. Rev. Lett. 65, 1379 (1990).

[13] R. Frésard, M. Dzierzawa, and P. Wölfle, Europhys. Lett. 15, 325 (1991).

[14] W. Zimmermann, R. Frésard, and P. Wölfle, Phys. Rev. B 56, 10097 (1997).

[15] A. Tandon, Z. Wang, and G. Kotliar, Phys. Rev. Lett. 83, 2046 (1999).

[16] G. Seibold, E. Sigmund, and V. Hizhnyakov, Phys. Rev. B 57, 6937 (1998).

[17] R. Frésard and G. Kotliar, Phys. Rev. B 56, 12909 (1997)

[18] H. Hasegawa, J. Phys. Soc. Jpn. 66, 1391 (1997); cond-mat/0005271.

[19] M. Lavagna, Phys. Rev. B 41, 142 (1990).

[20] Y. Okimoto, T. Katsufuji, Y. Okada, T. Arima, and Y. Tokura, Phys. Rev. B 51, 9581 (1995).

[21] P. Coleman, Phys. Rev. B 35, 5072 (1987).

[22] N. Read and D. M. Newns, J. Phys. C 16, 3273 (1983).

[23] E. Arrigoni, C. Castellani, M. Grilli, R. Raimondi, and G. C. Strinati, Phys. Rep. 241, 291 (1994).

[24] L. Gehlhoff, and R. Frésard, J. Phys: Condens. Matter, 8, L13 (1996).

[25] J. Kroha, P. J. Hirschfeld, K. A. Muttalib, and P. Wölfle, Solid State Commun. 83, 1003, (1992); J. Kroha, P. Wölfle, and T. A. Costi, Phys. Rev. Lett. 79, 261 (1997); J. Kroha, and P. Wölfle, Acta Phys. Pol. B 29 (12), 3781 (1998).

[26] J. E. Hirsch, and R. M. Fye, Phys. Rev. Lett. 56, 2521 (1986).

[27] N. E. Bickers, Rev. Mod. Phys. 59845 (1987).

[28] N. E. Bickers, Ph.D. thesis, (Cornell University) (1986).

[29] P. W. Anderson, Phys. Rev. 110 827(1958); Phys. Rev. 112 1900(1958).

[30] S. F. Edwards, and Y. V. Gulyaev, Proc. Royal Phys. Soc. A 279, 229 (1964); K. Ito, Mem. Amer. Math. Soc 4 , 1951.

[31] P. Coleman, Phys. Rev. B 29, 3035 (1984).

[32] A connected path is a temporal succession of $r$-values in which an initial value of $r$ equal to 1 at a site is continuously "transported" through the lattice until the final time step. For example, a "transport process" for which the value of $r_{j}$ at site $j$ jumps from 0 at time step $n$ to 1 at time step $(n+1)$ is valid only when it is connected to - via a matrix element $t_{i, j}$ - a site $i$ where the value of $r_{i}$ jumps from 1 at time step $(n+1)$ to 0 at time step $(n+2)$. For disconnected paths such jumps are not related, and they are excluded since such paths cannot generate "complete chains" (see previous section).

[33] D. Vollhardt, N. Blümer, K. Held, M. Kollar, J. Schlipf, M. Ulmke, and J. Wahle, Z. Phys. B 103, 283 (1997).

[34] For the atomic limit, this third term in Eq. (32) with $W \rightarrow 0$ in fact is equal to the contribution from the state with double occupancy for zero on-site interaction energy. However, this observation does not imply that we would have the option to recover the free limit without on-site interactions for an itinerant model by applying $W \rightarrow 0$. The phase relation between the various local states would still be missing. Therefore, the third term in Eq. (32) is spurious and we always have to take the valid limit $W \rightarrow \infty$.

[35] The original Kotliar and Ruckenstein representation has been introduced for problems with finite on-site interaction. In that case an additional field representing double occupancy needs to be introduced. Direct, but lengthy, calculations show that this field needs to be complex in order to get the correct result for the partition sum and correlation functions in the atomic limit.

[36] Th. Jolicœur, and J. C. Le Guillou, Phys. Rev. B 44, 2403 (1991). 
[37] Y. Bang, C. Castellani, M. Grilli, G. Kotliar, R. Raimondi, and Z. Wang, Proceedings of the Adriatico Research Conference and Miniworkshop "Strongly Correlated Electron Systems III", Eds. G. Baskaran, A. E. Ruckenstein, E. Tosatti et Yu Lu, Int. J. of Mod. Phys. B 6, 531 (1992). 
Fig. 1

$Z_{\text {at }}=$

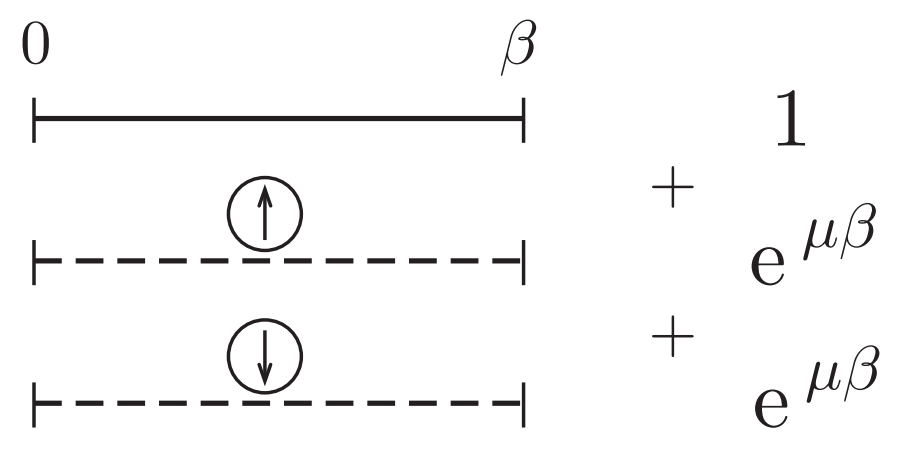




\section{Fig. 2}
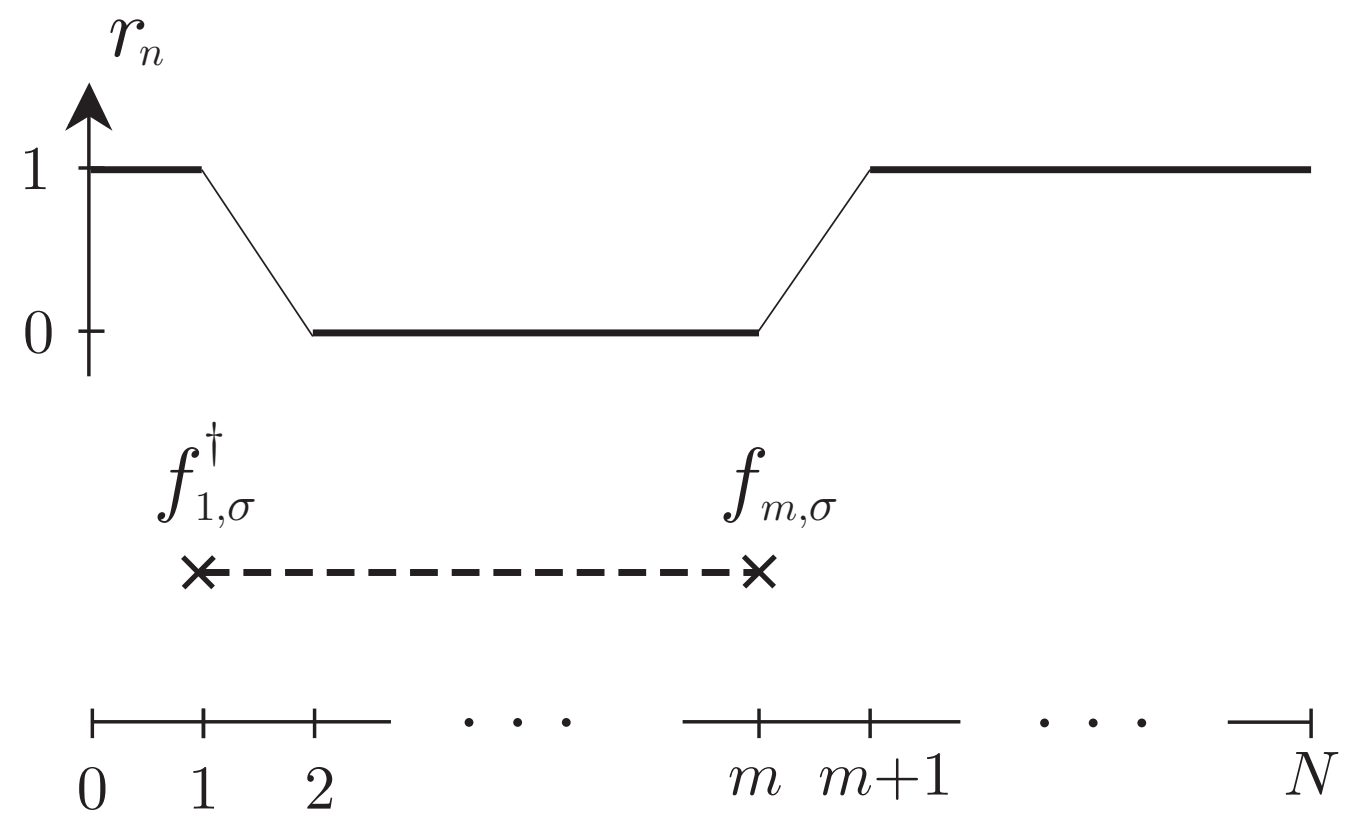